\title{
Tratamiento con 1,25 dihidroxicolecalciferol (DHCC) en niños con diferentes formas de raquitismo resistente
}

Dres. Martha Eggers M. $y$ Francisco Beas F.l

\author{
Treatment with 1.25 Dihydroxicholecalcipherol in Vitamin D resistant rickets
}

\begin{abstract}
The respomse of different tonus of Yitamin $\mathrm{D}$ rassistant rickets to 1,25 Dihydroxicholecalcipherol Has studied.

Eight patients with isolated hypophosphersia showed good clinical response with partial radiographic resolution, diminution of serum alcaline phosphatuse and persistence of hipephesphemia.

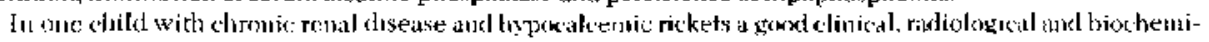
tal responst wars ulmerved.
\end{abstract}

\section{INTRODUCCION}

El tratamiento del racuitismo resistente a la vitamina $D$ es de difícil manejo. Habitualmente es necesario someter a los pacientes a dosis farmacológicas de vitamina $\mathbf{D}$, con el fin de lograr resultados terapéuticos (a nivel óseo y en lo posible metabólico). Es aceptado hoy en día que la vitamina

IInstituto de Investigaciones Clinicas, Undad de Enducrinologîa y Genébica Intantil y Departamento de Pediatria y Cinıgia Intiantil, Hospinal Paula Jaraquenada, Facultad de Medicima, División Sur, Universidad de Chile.
D debería ser considerada como una prohormona del 1.25 Dihidroxicolecalciferol (DHCC), principal metabolito activo, el que suministrado en dosis fisiológica deberia producir respuesta terapéutica satisfactoria, sin efectos colaterales.

El presente trabajo muestra la experiencia clínica obtenida en un grupo de pacientes con raquitismo vitamino-D-resistente, hipofósfémico simple (familiar o esporádico), tratados con 1,25 DHCC. Comparativamente se estudió la respuesta terapéutica en pacientes con raquitismo hipocalcémico, vitamino-D-dependiente (Raquitismo de Prader) y en un caso con raquitismo por insuficiencia renal crónica. 


\section{MATERIAL Y METODO}

El estudio se efectuó en 12 pacientes cuya distribución por diagnóstico, edad y sexo se ve en la Tabla 1.

En los pacientes en tratamiento previo con vita- mina D2 se suspendí ésta por un mínimo de I mes o hasta recaída bioquimica de actividad ranquitica, con o sin alteración radiológica. La dosis promedio usada de DHCC y la duración del tratamiento están consignadas en la Tabla 1. En todos los pacientes se efectuó:

Tabla 1

Pacientes tratados con DHCC: diagnóstico, edad, sexo, dosis promedio y duración del tratamiento.

\begin{tabular}{|c|c|c|c|c|c|}
\hline $\begin{array}{l}\text { Tipo de } \\
\text { Raquitismo }\end{array}$ & $\begin{array}{c}\text { Paciente } \\
\text { N. }\end{array}$ & Edad & Sexo & $\begin{array}{l}\text { Dosis Prom. } \\
\mu \mathrm{g} / \mathrm{kg} \text {-día }\end{array}$ & $\begin{array}{l}\text { Duración } \\
\text { Trat Meses }\end{array}$ \\
\hline $\begin{array}{l}\text { Hipocalcémico } \\
\text { vit D-depen- } \\
\text { diente }\end{array}$ & $\begin{array}{l}1 \\
2 \\
3\end{array}$ & $\begin{array}{c}5 a \\
14 a 3 m \\
4 a\end{array}$ & $\begin{array}{l}\mathbf{M} \\
\mathbf{M} \\
\mathbf{F}\end{array}$ & $\begin{array}{l}0,07 \\
0,06 \\
0,08\end{array}$ & $\begin{array}{c}8 \\
8,5 \\
6\end{array}$ \\
\hline $\begin{array}{l}\text { Por insuficierticia } \\
\text { renal crónica }\end{array}$ & 4 & $6 a$ & $F$ & 0,04 & 3 \\
\hline $\begin{array}{l}\text { Hipofosfémico } \\
\text { simple (familiar } \\
\text { o esporídico) }\end{array}$ & $\begin{array}{r}5 \\
6 \\
7 \\
8 \\
9 \\
10 \\
11 \\
12\end{array}$ & $\begin{array}{l}6 \text { a } 7 \mathrm{~m} \\
2 \text { a } 9 \mathrm{~m} \\
2 \text { a } 11 \mathrm{~m} \\
15 \mathrm{a} \\
15 \mathrm{a} \\
11 \mathrm{a} \\
1 \mathrm{a} 8 \mathrm{~m} \\
6 \mathrm{a} 3 \mathrm{~m}\end{array}$ & $\begin{array}{l}F \\
F \\
F \\
M \\
F \\
M \\
F \\
M\end{array}$ & $\begin{array}{l}0,06 \\
0,08 \\
0,08 \\
0,03 \\
0,06 \\
0,08 \\
0,10 \\
0,10\end{array}$ & $\begin{array}{r}9 \\
8 \\
11 \\
6 \\
5 \\
6 \\
4 \\
3\end{array}$ \\
\hline
\end{tabular}

a) Estudio clínico: anamnesis personal y familiar, examen físico y control clínico mensual.

b) Estudio bioquimico: calcemia, fosfemia, fosfatasas alcalinas y calciuria inicial y mensual.

c) Estudio radiológico: radiografía de carpo para evaluar signos raquíticos y edad osea antes y después de 3 y 6 meses de tratamiento.

\section{RESULTADOS}

1. Raquitismo vitamino-D-dependiente. En la Tabla 2 pueden observarse los resultados obtenidos en este tipo de pacientes, desde el punto de vista clínico, bioquímico y radiológico, al usar dosis de DHCC de 0,06 - 0,08 $\mu \mathrm{g} / \mathrm{kg} /$ día.

1. Respuesta clínica: ritmo de crecimiento superior al promedio normal para la edad en los 3 pacientes. Desaparición de los dolores óseos y de la fatigabilidad fácil en los que la presentaban.

2. Respuesta bioquímica: la calcemia se mantuvo dentro de límites normales en 2, persistiendo discreta hipocalcemia en 1. La fosfemia fluctuó entre valores bajos y normales. Las fosfatasas alca- linas tendieron a normalizarse, persistiendo valores discretamente altos en 1 de los pacientes después de 6 meses de tratamiento. La calciuria se mantuvo baja en 2 y en 1 subió a un valor máximo entre 8 - $10 \mu \mathrm{g} / \mathrm{kg} /$ día.

3. Respuesta radiológica: en la paciente 3 hubo buena reparación de las lesiones raquíticas iniciales, en el paciente 2 persistieron lesiones mínimas y el paciente 1 no presentó lesiones raquiticas durante el tratamiento.

II. Raqujtismo asociado a insuficiencia renal crónica. Se trató con dosis bajas de DHCC, observándose una respuesta buena, resumida en la Tabla 2 .

1. Respuesta clínica: creció $0,66 \mathrm{~cm} / \mathrm{mes}$, lo que en parte podría también deberse al tratamiento simultáneo para corregir la acidosis y la retención nitrogenada. Los dolores óseos y la fatigabilidad fácil disminuyeron en forma notoria.

2. Respuesta bioquimica: las fosfatasas alcalinas se normalizaron a los 3 meses de tratamiento. La hipocalcemia inicial se corrigio. La fosfemia y la calciuria se mantuvieron en limites aceptables. 
Respuesta clínica, bioquínica y cadiologica al tratamiento con DHCC.

Raquitismo vit $\mathrm{D}$ dependiente

Hayuitismo asociado

a Insuf. renal

\begin{tabular}{lcccc}
\hline Paciente N. & 1 & 2 & 3 & 4 \\
Edad & 5 años & 11 a $3 \mathrm{~m}$ & 4 años & 6 años \\
Dosis $\mu \mathrm{g} / \mathrm{kg} / \mathrm{día}$ & 0,07 & 0,06 & 0,06 & 0,04
\end{tabular}

\begin{tabular}{|c|c|c|c|c|c|}
\hline $\begin{array}{l}\text { Signos } \\
\text { clinicos }\end{array}$ & $\begin{array}{l}\text { Crecimiento } \\
\text { Dolores óseos } \\
\text { Fatigabilidad }\end{array}$ & $\begin{array}{l}0,75 \mathrm{~cm} / \mathrm{mes} \\
\text { Desaparecen } \\
\text { Desaparecen }\end{array}$ & $\begin{array}{l}1 \mathrm{~cm} / \text { mes } \\
\text { Desaparecen } \\
\text { Desaparecen }\end{array}$ & $\begin{array}{l}0,83 \mathrm{~cm} / \mathrm{mes} \\
\text { Desaparecen } \\
\text { Desaparecen }\end{array}$ & $\begin{array}{l}0,66 \mathrm{~cm} / \mathrm{mes} \\
\text { Disminuyen } \\
\text { Disminuyen }\end{array}$ \\
\hline \multirow[t]{3}{*}{$\begin{array}{l}\text { Parámetros } \\
\text { bioquímicos }\end{array}$} & $\begin{array}{l}\text { Calcemia inicial } \\
\text { Calcemia con trat. }\end{array}$ & $\begin{array}{l}8.9 \mathrm{mg} \% \\
8.7 \mathrm{mg} \%\end{array}$ & $\begin{array}{l}8.5 \mathrm{mg} \% \\
8.1 \mathrm{mg} \%\end{array}$ & $\begin{array}{l}7.4 \mathrm{mg} \% \\
7.8 \mathrm{mg} \%\end{array}$ & $\begin{array}{l}7.04 \mathrm{mg} \% \\
8.79 \mathrm{mg} \%\end{array}$ \\
\hline & $\begin{array}{l}\text { Fosfemia inicial } \\
\text { Fosfemia con trat. } \\
\text { Fosf. Alc. inicial } \\
\text { Fosf. Alc. con trat. }\end{array}$ & $\begin{array}{l}2.3 \mathrm{mg} \% \\
4.5 \mathrm{mg} \% \\
1042 \mathrm{mUL} / \mathrm{ml} \\
563 \mathrm{mUL} / \mathrm{ml}\end{array}$ & $\begin{array}{l}4.5 \mathrm{mg} \% \\
4.0 \mathrm{mg} \% \\
1082 \mathrm{mUL} / \mathrm{ml} \\
599 \mathrm{mUL} / \mathrm{ml}\end{array}$ & $\begin{array}{l}3.0 \mathrm{mg} \% \\
3.6 \mathrm{mg} \% \\
1260 \mathrm{mUl} / \mathrm{ml} \\
623 \mathrm{mUL} / \mathrm{ml}\end{array}$ & $\begin{array}{l}4.36 \mathrm{mg} \% \\
5.57 \mathrm{mg} \% \\
880 \mathrm{mUl} / \mathrm{ml} \\
333 \mathrm{mUl} / \mathrm{ml}\end{array}$ \\
\hline & $\begin{array}{l}\text { Calciuria inicial } \\
\text { Calciuria con trat. }\end{array}$ & $\begin{array}{l}2.9 \mathrm{mg} / \mathrm{kg} / \mathrm{día} \\
8.0 \mathrm{mg} / \mathrm{kg} / \mathrm{día}\end{array}$ & $\begin{array}{l}1.0 \mathrm{mg} / \mathrm{kg} / \mathrm{dia} \\
1.0 \mathrm{mg} / \mathrm{kg} / \mathrm{dia}\end{array}$ & $\begin{array}{l}1.4 \mathrm{mg} / \mathrm{kg} / \mathrm{dia} \\
1.0 \mathrm{mg} / \mathrm{kg} / \mathrm{dia}\end{array}$ & $\begin{array}{r}2.3 \mathrm{mg} / \mathrm{kg} / \mathrm{día} \\
4.0 \mathrm{mg} / \mathrm{kg} / \mathrm{día}\end{array}$ \\
\hline $\begin{array}{l}\text { Evolución } \\
\text { radiológica }\end{array}$ & Signos raquíticos & Reparados & Reparados & Mínimos & Reparados \\
\hline
\end{tabular}

Tabla 3

Respuesta clinica, bioquímica y radiológiea al tratamiento con DHCC y fosfato oral en 8 casos de hipofosfemia simple.

\begin{tabular}{|c|c|c|c|c|c|c|c|c|c|}
\hline & $\begin{array}{l}\text { Caso N. } \\
\text { Edad } \\
\text { Dosis } \mu g / k g / d i ́ a\end{array}$ & $\begin{array}{c}5 \\
6 \text { a } 7 \mathrm{~m} \\
0.06\end{array}$ & $\begin{array}{c}6 \\
2 \mathrm{a} 9 \mathrm{~m} \\
0.08\end{array}$ & $\begin{array}{c}7 \\
2 \mathrm{a} 11 \mathrm{~m} \\
0.08\end{array}$ & $\begin{array}{c}8 \\
15 \mathrm{a} \\
0.03\end{array}$ & $\begin{array}{c}9 \\
15 \mathrm{a} \\
0.06\end{array}$ & $\begin{array}{c}10 \\
11 \mathrm{a} \\
0.08\end{array}$ & $\begin{array}{c}11 \\
1 \mathrm{a} 8 \mathrm{~m} \\
0.10\end{array}$ & $\begin{array}{c}12 \\
6 \mathrm{a} 3 \mathrm{~m} \\
0.10\end{array}$ \\
\hline $\begin{array}{l}\text { Signos } \\
\text { ánicos }\end{array}$ & $\begin{array}{l}\text { Crecimiento } \\
\text { Dolores oseos } \\
\text { Fatigabilidad }\end{array}$ & $\begin{array}{l}0,55 / \text { mes } \\
\text { Besapar. } \\
\text { Desapar. }\end{array}$ & $\begin{array}{l}0,55 / \text { mes } \\
\text { Desapar. } \\
\text { Desapar. }\end{array}$ & $\begin{array}{l}\text { 0,36/mes } \\
\text { Desapar. } \\
\text { Desapar. }\end{array}$ & $\begin{array}{l}0,33 / \text { mes } \\
\text { Desapar. } \\
\text { Desapar. }\end{array}$ & $\begin{array}{l}0,25 / \text { mes } \\
\text { Desapar. } \\
\text { Desapar. }\end{array}$ & $\begin{array}{l}0,50 / \text { mes } \\
\text { Desapar. } \\
\text { Dismin. }\end{array}$ & $\begin{array}{l}0,80 / \text { mes } \\
\text { Desapar. } \\
\text { Dismin. }\end{array}$ & $\begin{array}{l}0,40 / \text { mes } \\
\text { Desapar. } \\
\text { Dismin. }\end{array}$ \\
\hline $\begin{array}{l}\text { Pará- } \\
\text { netros }\end{array}$ & $\begin{array}{l}\text { Calcemia inicial } \\
(\mathrm{mg} \%) \text { " a los }\end{array}$ & 9.3 & 9.6 & 9.1 & 8.4 & 10.6 & 7.1 & 8.6 & 7.7 \\
\hline \multirow{7}{*}{$\begin{array}{l}\text { bioqui- } \\
\text { micos }\end{array}$} & 6 meses & 8.9 & 8.8 & 9.8 & 7.1 & 8.2 & 9.6 & $9.0(4 \mathrm{~ms})$ & $8.2(3 \mathrm{~ms})$ \\
\hline & $\begin{array}{l}\text { Fosfemia inicial } \\
\text { (mg \%)" a los }\end{array}$ & 1.6 & 2.2 & 2.0 & 2.1 & 1.3 & 2.1 & 2.9 & 1.9 \\
\hline & 6 meses & 2.6 & 2.0 & 2.1 & 2.1 & 2.3 & 3.5 & $2.0(4 \mathrm{~ms})$ & $2.2(3 \mathrm{~ms})$ \\
\hline & $\begin{array}{l}\text { Fosf. Alc. inic. } \\
\text { (m UI/ml)" a los }\end{array}$ & 294 & 1604 & 2049 & 645 & 995 & 1589 & 1721 & 684 \\
\hline & 6 meses & 683 & 796 & 881 & 385 & 892 & 924 & 1211 (4 ms) & 590 (3 ms) \\
\hline & $\begin{array}{l}\text { Cakiuna inic: } \\
\text { (mg \%)" a los }\end{array}$ & 4.0 & 1.8 & 3.2 & 2.6 & 1.3 & 2.0 & 1.1 & 1.0 \\
\hline & 6 meses & 2.5 & 2.2 & 5.0 & 4.7 & 1.0 & 2.0 & 2.0 (4 ms) & $1.0(3 \mathrm{~ms})$ \\
\hline
\end{tabular}

Estudio Reparación lenta y parcial, no total, de las lesiones raquiticas radioló- Retardo edad ósea discreto a moderado 
3. Respuesta radiológica: fue rápida, apreciándose a las 6 semanas de tratamiento una reparación de las imágenes quísticas y una mejor mineralización del hueso.

III. Raquitismo hipofosfếmico. Se usó dosis en rango fisiológico alto en 6 pacientes y en 2 se usó dosis ligeramente mayores. La respuesta al tratamiento se resume en la Tabla 3.

1. Respuesta clínica: el crecimiento fue normal en $6, y$ en 2 fue deficiente, pero en éstos hay que destacar que presentaban deformación severa de extremidades inferiores, lo que dificulta una evaJuación adecuada.

2. Respuesta bioquímica: la calcemia se mantuvo en rango normal en todos. La hipofosfemia persistió sin modificaciones. Las fosfatasas alcalinas descendieron en forma parcial, normalizándose sólo en un paciente de 15 años. La calciuria aumentó, pero dentro de límites aceptables.
3. Respuesta radiológica: hubo reparación aceptable pero lenta, después de varios meses de tratamiento. El retardo de la edad ósea persistió, pero menos acentuado.

\section{COMENTARIO Y DISCUSION}

Se denomina raquitismo resistente a aquel que no responde a la dosis habituales de vitamina D. Las causas pueden ser muy variadas, y el tratamiento debe siempre ir enfocado de acuerdo con la patología de base o en su defecto, a tratar de corregir las alteraciones metabólicas.

Las causas de raquitismo podrían ser divididas en 2 grupos:

1. Con alteración primaria o secundaria en el metabolismo de la vitamina $\mathbf{D}$.

2. Con pérdida exagerada de fosfatos por el rinón, son las tubulopatías que condicionan una hipofosfemia. Ver Tabla 4.

Tabla 4

Causas de raquitismo

Falta de aporte

1. Por déficit de vit. D: Síntesis endúgena o disminuida

Malabsorción intestinal

II. Por alteración en el metabolismo de la vit. $\mathbf{D}$

1. Alteración en la hidroxilación hepática: anticonvulsionantes, enfermedad hepatica

2. Alteración en la hidroxilación renal: déficit $1 \times 25$ hidroxilasa renal

A. Congénita : raquitismo vit. D-dependiente

B. Adquirido : daño renal crónico, hipoparatimidisma, pseudohipoparatiroidismo

III. Tubolopatias : fosfaturia aumentada e hipolosfemia

1. Hipofosfemia simple, familiar o esporádica

2. Síndrome de Fanconi : idiopátieo, tirosinosis

3. Acidosis tubular distal

4. Cistinosis

5. Tumores racuitugénicos

La vitamina $D$ puede ser considerada como una prohormona que da origen a varios metabolitos activos que intervienen en el metabolismo fosfocálicico. De éstos el principal sería el 1,25 dihidroxicolecalciferol de acuerdo a numerosas investigaciones experimentales y clínicas. ${ }^{1-5,{ }^{18}}$ Este metabolito cumple con ciertos requisitos que permitirían considerarlo como una hormona: sólo se sintetiza a nivel del riñón, pasa a la circulación y ejerce su acción sobre distintos tejidos: intestino, riñón y hueso $y$ cuenta con mecanismos propios que regulan sus sintesis. Ver Cuadro 1. 
Cuadro 1

Metabolismo de la vitamina $\mathbf{D}$

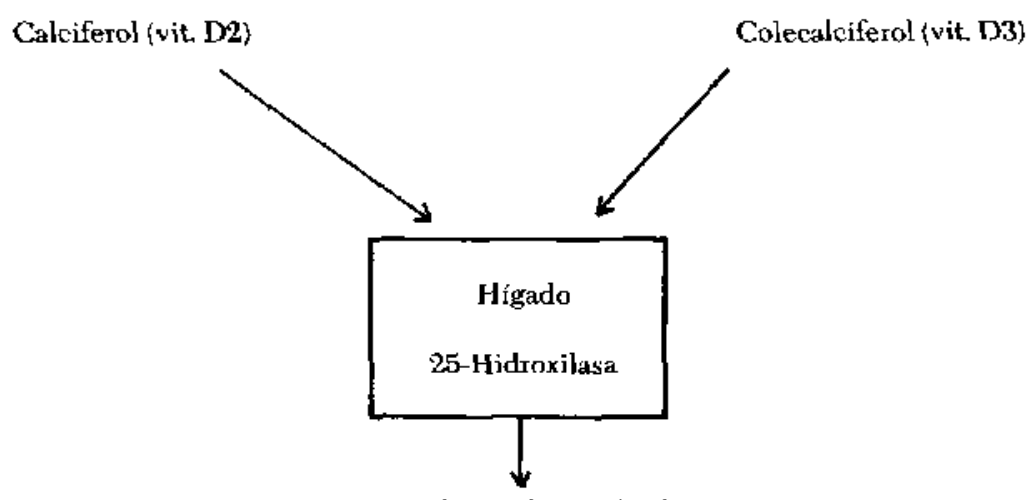

25-Hidroxicolecalciferol

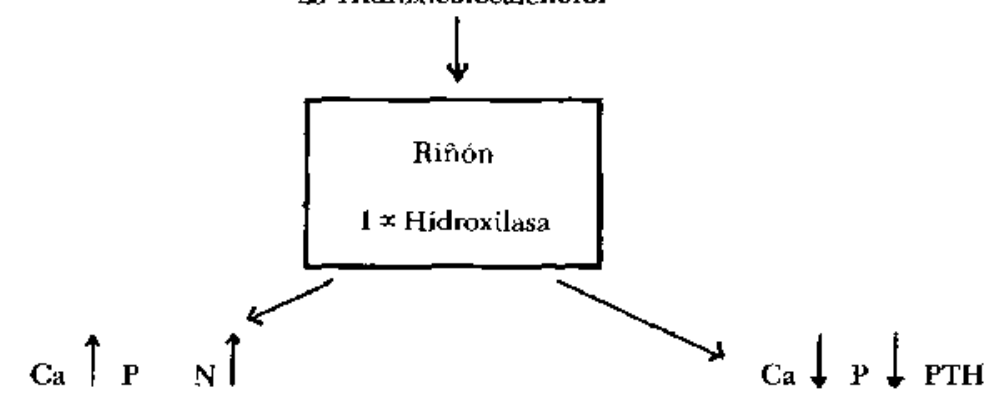

$24,25(\mathrm{OH}) 2-\mathrm{D} 3$

1,25 Dihidroxicolecalciferol

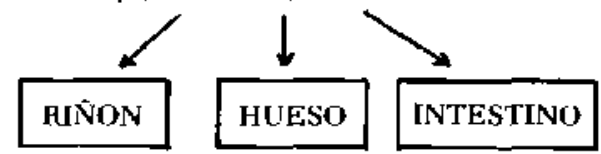

En los raquitismos resistentes debidos a una alteración primaria o secundaria de la vitamina $\mathrm{D}$, está indicado el uso de la vitamina $\mathrm{D}$ o de sus metabolitos. $^{7,19}$

El raquitismo vitamino-D-dependiente corresponde al raquitismo pseudocarencial descrito por Prader. ${ }^{8}$ Se debería a un defecto enzimático congénito con déficit de la $I \propto$ hidroxilasa renal, necesaria para la conversión del 25 hidroxicolecalciferol a 1,25 dihidroxicolecalciferol. ${ }^{90}$ Se caracteriza por raquitismo de comienzo precoz, con hipocalcemia difícil de corregir. Se ha comunicado que responden bien al tratamiento ya sea con dosis farmacológicas de vitamina D2 o con dosis fisiologicas del metabolito $1 \times$ hidroxilado, lográndose la corrección clínica, bioquímica y radiológica. Al suspender el tratamiento reaparece el cuadro." En nuestros 3 pacientes cuyo cuadro clínico y evolución concuerdan con esta forma de raquitismo, pudimos apreciar una buena respuesta usando dosis en rango fisiológico, destacando la aceleración del crecimiento.

En la insuficiencia renal cronica hay una disminución del parénquima renal funcionante con déficit parcial de la $1 \propto$ hidroxilasa renal, por lo que disminuye la síntesis total de 1,25 DHCC. ${ }^{2,}$, 
13. 20 Este déficit junto a otros factores de tipo metabólico impiden una adecuada mineralización ósea. En nuestra paciente estudiada observamos una repuesta buena y rápida, tanto clínica, bioquímica como radiológica, con dosis bajas de DHCC.

En los raquitismos asociados a falla tubular con pérdida exagerada de fosfatos, no se ha demostrado que exista una alteración cuantitativa en el metabolismo de la vitamina $\mathrm{D}$, al menos no en la hipofosfemia simple, que es la forma más frecuente. $^{14}$ La hipofosfemia simple, familiar o esporádica, puede producir raquitismo de comienzo precoz o tardio, con retardo de crecimiento y deformaciones óseas progresivas, especialmente de extremidades inferiores. Estos pacientes responden relativamente bien al tratamiento con fosfato oral asociado a dosis altas de vitamina D2, 15, 16, 17, 21 En nuestros 8 pacientes tratados con DHCC observamos una respuesta favorable, aunque lenta, con dosis fisiológicas, lo que no se logra con dosis equivalentes de vitami. na D2.

De los resultados obtenidos en los pacientes estudiados queremos destacar los siguientes hechos:

1. Fenómeno de "catch up growth" en los 3 pacientes con raquitismo vitamino- $D$ dependiente.

En la paciente con insuficiencia renal crónica se observó crecimiento bueno durante los 3 meses que fué tratada, reconocemos sí que es un periodo muy corto para evaluar crecimiento.

En la hipofosfemia simple el ritmo de crecimiento se normalizó en 6 de los 8 pacientes tratados.

2. La sintomatología de dolores óseos y la fatigabilidad fácil mejoró en todos los pacientes que la presentaban, independiente del diagnóstico.

3. La respuesta bioquímica fue rápida en la insuficiencia renal, normalizándose la calcemia y las fosfatasas alcalinas a los 3 meses de tratamiento. La reparación de las lesiones radiográficas fue rápida, a las 6 semanas de tratamiento.

En el raquitismo vitamino-D-dependiente la respuesta bioquímica y radiológica también fue satisfactoria, aunque más lenta.

En el raquitismo hipofosfémico simple el descenso de las fosfatasas alcalinas fue lento y parcial, sin llegar a rango normal. La calciuria aumentó en forma gradual, sin sobrepasar limite razonable ( 8 $\mathrm{m} / \mathrm{kg} / \mathrm{dia}$ ) excepto en 1 de los pacientes tratados.
La recuperación radiológica en este grupo fue también lenta y parcial, excepto en 1 paciente tratado con dosis algo mayor, DHCC 0,10 $\mu \mathrm{g} / \mathrm{kg} /$ dia, en el que hubo reparación radiológica a los 2 meses de tratamiento.

Las observaciones clínicas obtenidas a través de los casos mencionados nos podrian permitir concluir lo siguiente:

1. En el Haquitismo Hipocalcémico o Vitamina D dependiente, la ventaja del 1,25 DHCC sobre la vitamina $D$ es evidente, porque bastaron dosis fisiológicas en rango alto $(0,8 \mu \mathrm{g} / \mathrm{kg} /$ día $)$ para normalizar el cuadro clínico, bioquímico y radiografico. Esto no se consigue con la vitamina $D$ sino a dosis farmacológicas muy altas, con los riesgos consiguientes.

2. En el Raquitismo asociado a Insuficiencia renal crónica, parecen ser suficientes dosis fisiológicas bajas $(0,38 \mu \mathrm{g} / \mathrm{kg} /$ dia en el caso tratrado).

3. En el Raquitismo Hipofosfémico simple (tanto familiar como esporádico) la dosis óptima parece estar al iniciar el tratamiento, en 0,10 $\mu \mathrm{g} / \mathrm{kg} /$ día, empleada en las casos 11 y 12 . Las dosis menores empleadas en este estudio en los otros 6 casos no permitieron una buena respuesta bioquímica ni radiográfica, aunque sí una buena respuesta clínica, porque el crecimiento se reanuda a ritmo nornal.

4. El uso exclusivo de fosfato oral en el Raquitismo Hipofosfémico simple (indicado en los casos $3,4,5,7$ y 8 ) se demostró insuficiente, como terapia exclusiva, porque el Sindrome Raquítico persiste o recae.

\section{RESUMEN}

Se estudió la respuesta al tratamiento con 1,25 DHCC en distintas formas de raquitismo resistente.

En 8 pacientes con hipofosfemia simple hubo buena respuesta clínica, reparación radiológica parcial, disminución de fosfatasas alcalinas y persistencia de hipofosfemia.

En el raquitismo hipocalcémico y en un paciente con insuficiencia renal crónica hubo buena respuesta clínica, radiológica y bioquímica.

\section{REFERENCIAS}

1Noman, A. W.: D. metabolisin and Ca absorption. An. J. Med. 67:6: $989,1979$.

2Roat, A. Hamison, H. Recent andvances in Ca metab. The J. of Fed. 83,$2 ; 177,1976$. 
3Kodicek, E. The story al Vit. D from Vit. to homonte. Lencet, I. itri35, 1974.

${ }^{4}$ Holick, De Luca. : Vit. D ntetaholism. Aur. Hev. Med. 25: 344, 1974.

${ }^{5}$ De Luan, $H . F_{*}$ Vit. D. The Am. J. of Med. Vol. $57 /$, VII 1974.

${ }^{6}$ Beales, Char, Oldhan, De Luca: Vit. D. Pediatrics. Vol. 57, N. ${ }^{\circ} \overline{2}$, $\checkmark 1976$.

'Prader, A.: Vit. D. Helvetica Ped. Acta, Vol, 30, N."2, VII I975. SPrader, A. IJing, R., Hejeri, E., Eine Besondere: Froul der primaren Vit, resistenten Bachitis init Hypocalcemics. Helv. Ped. Actu. I6: 452, 1961 .

Froser, Kooh, Kind, Holfck, Tamaka, De Lwea: Pathogenesis of hered. Vit. D Dep. Rickets. New. EngJ, J. of Med. 269: 817 . 1973.

${ }^{10}$ Amaud, Majer, Reade, Scriver, Whelan.: Vit. $\mathrm{D}$ dependency. Pediatries. 46; 871, 1970.

IIReode, Scriver, Glonewx, Nogrady, Delvin, Poirler, Hollick, De Luar: Kesponde to $J \times$ hydroxi Vit. $D 3$ in Vit. D dependency. Ped. Res. 9: 5:33, 1975 .

12Erickman, Cobum, Masy, Norman, 1,25 Dihydroxi Vit. D3 in numnal man and in patients with serabl failure. An. Int. Med. 40. 161,1974
13Henderson, Russell, Letingham, Smith, Otiver, Walton, Small Preston. Wamer, Noman. : Effects of 1,25 DHCC in chronic renal failıre. Lancet i. $379,1974$.

14Brickonan, Cobum, Kurokowa, Bethune, Harrison, Norman.: Actions of 1,25 DHCC. in patients wijth hymphosphatemic: $V$ it. I) resistent rickets.

15West, Blanton, Sütueman, Holkand: Use of phosphate 5 al ts als anl adjunct to Vit. D in treatment of hypophosphatemic Vit D retrisctory rickets. The J. of Ped. Vol. 64. N." 4, IV 1964.

Itischoen, Reynodd: Severe hypophosphatemic ricket;. Am. J. Dis. Child. Vol. 120 , VII 1970.

17Frame, Smith, Fleury, Morson,: Oral Phosphate in Vit. D refractory rickets. Am. J. of Chilid. Vol. lOS, VIII l\$63.

${ }^{18}$ De Luca, H. F.: The Vit D system in the regulation of Ca and $P$ tretab. Nuts. Rev, Vol. 37, N.'6, p. 161, 1979.

19 Balson, Garobedian, Sorgriard, Hohck, De Luoa: 1,25 DHCC and $1 \times$ OH D3 in Children. Ped. Res. 9: 586, 1875

${ }^{20} \mathrm{Ch}$ an, De Luca; Chronic renal fail ure and treatment with Calcitrial. J. Am. Med. Assoc. 241/12, p. 1242, 1979.

21 Chesney, Mozedd, Rose.: Suprauournal 25 OH D3 and Subnonmal 1,25 DIJCC. Tlevir role in X-linked hypophosphutemle rickets. Am. J, Dis, Child, 134/2; 141), 1940. 